\title{
Mechanism of Preconditioning by Isoflurane in Rabbits: A Direct Role for Reactive Oxygen Species
}

Katsuya Tanaka, M.D., , Dorothee Weihrauch, D.V.M. Ph.D.,† Franz Kehl, M.D. D.E.A.A., ${ }^{*}$ Lynda M. Ludwig, B.S., $\ddagger$ John F. LaDisa, Jr., M.S. „‡ Judy R. Kersten, M.D.,§ Paul S. Pagel, M.D. Ph.D. „| David C. Warltier, M.D. Ph.D.\#

\begin{abstract}
Background: Reactive oxygen species (ROS) contribute to myocardial protection during ischemic preconditioning, but the role of the ROS in protection against ischemic injury produced by volatile anesthetics has only recently been explored. We tested the hypothesis that ROS mediate isoflurane-induced preconditioning in vivo.

Methods: Pentobarbital-anesthetized rabbits were instrumented for measurement of hemodynamics and were subjected to a $30 \mathrm{~min}$ coronary artery occlusion followed by $3 \mathrm{~h}$ reperfusion. Rabbits were randomly assigned to receive vehicle ( $0.9 \%$ saline), or the ROS scavengers $\mathrm{N}$-acetylcysteine (NAC; $150 \mathrm{mg} / \mathrm{kg}$ ) or $\mathrm{N}-2$ mercaptopropionyl glycine (2-MPG; $1 \mathrm{mg} \cdot \mathrm{kg}^{-1} \cdot \mathrm{min}^{-1}$ ), in the presence or absence of 1.0 minimum alveolar concentration (MAC) isoflurane. Isoflurane was administered for $30 \mathrm{~min}$ and then discontinued $15 \mathrm{~min}$ before coronary artery occlusion. A fluorescent probe for superoxide anion production (dihydroethidium, $2 \mathrm{mg}$ ) was administered in the absence of the volatile anesthetic or $5 \mathrm{~min}$ before exposure to isoflurane in $\mathbf{2}$ additional groups $(n=8)$. Myocardial infarct size and superoxide anion production were assessed using triphenyltetrazolium staining and confocal fluorescence microscopy, respectively.

Results: Isoflurane $(P<0.05)$ decreased infarct size to $24 \pm 4 \%$ (mean \pm SEM; $n=10$ ) of the left ventricular area at risk compared with control experiments $(43 \pm 3 \% ; n=8)$. NAC $(43 \pm 3 \% ; n=7)$ and 2-MPG ( $42 \pm 5 \% ; n=8)$ abolished this beneficial effect, but had no effect on myocardial infarct size $(47 \pm 3 \% ; n=8$ and $46 \pm 3 ; \mathbf{n}=7$, respectively) when administered alone. Isoflurane increased superoxide anion production as compared with control experiments ( $28 \pm 12$ vs. $-6 \pm 9$ fluorescence units; $P<0.05)$.

Conclusions: The results indicate that ROS produced following administration of isoflurane contribute to protection against myocardial infarction in vivo.
\end{abstract}

LARGE quantities of reactive oxygen species (ROS) released during reperfusion after coronary artery occlusion damage proteins responsible for intracellular homeostasis, produce tissue injury, ${ }^{1-3}$ depress contractile function, and increase myocardial infarct size. In contrast, small quantities of ROS may exert beneficial effects during ischemia and reperfusion when released before a prolonged ischemic event. ${ }^{4}$ ROS derived from mitochon-

\footnotetext{
* Research Fellow, † Assistant Professor, ‡ Graduate Student, $₫$ Professor, Department of Anesthesiology, Pharmacology and Toxicology, $\|$ Professor and Director of Cardiac Anesthesia, \# Professor, Department of Anesthesiology, Pharmacology and Toxicology, and Medicine (Division of Cardiovascular Diseases), and Vice Chairman for Research, Department of Anesthesiology.

Received from the Departments of Anesthesiology, Pharmacology and Toxicology, and Medicine (Division of Cardiovascular Diseases), the Medical College of Wisconsin, and the Clement J. Zablocki Veterans Affairs Medical Center, Milwaukee, Wisconsin. Submitted for publication April 19, 2002. Accepted for publication July 10, 2002. Supported in part by grant Nos. HL 03690 (J.R.K.), HL 63705 (J.R.K.), HL 54820 (D.C.W.), and Anesthesiology Research Training Grant No. GM 08377 (D.C.W.) from the United States Public Health Service, National Institutes of Health, Bethesda, Maryland

Address reprint requests to Dr. Warltier: Medical College of Wisconsin, MEBM4280, 8701 Watertown Plank Road, Milwaukee, Wisconsin 53226. Address electronic mail to cknapp@mcw.edu. Individual article reprints maybe purchased through the Journal Web site, www.anesthesiology.org.
}

dria during a brief ischemic episode produce preconditioning. ${ }^{5,6}$ Free radical scavengers administered during ischemic preconditioning (IPC) markedly attenuate the protective effect of the preconditioning stimulus on infarct size. ${ }^{5,7}$ These data suggest that IPC is mediated in part by small quantities of ROS released during preconditioning. Volatile anesthetics protect myocardium against infarction through a signal transduction pathway that includes adenosine type 1 receptors, ${ }^{8-10}$ protein kinase $\mathrm{C},{ }^{9,11}$ inhibitory guanine regulatory proteins, ${ }^{12}$ and mitochondrial and sarcolemmal adenosine triphosphate-regulated potassium $\left(\mathrm{K}_{\mathrm{ATP}}\right)$ channels. ${ }^{13-16} \mathrm{~A}$ recent investigation by Müllenheim et al. provides compelling evidence that ROS also mediate myocardial protection produced by volatile anesthetics. ${ }^{17}$ We sought to confirm and extend these important results by examining the hypothesis that ROS scavengers inhibit isoflurane-induced protection against irreversible ischemic injury. We further tested the hypothesis that isoflurane directly generates ROS in rabbit ventricular myocardium in vivo using a confocal microscopic technique combined with the superoxide anion-specific fluorescent probe dihydroethidium.

\section{Methods}

All experimental procedures and protocols used in this investigation were reviewed and approved by the Animal Care and Use Committee of the Medical College of Wisconsin. Furthermore, all conformed to the Guiding Principles in the Care and Use of Animals of the American Physiologic Society and were in accordance with the Guide for the Care and Use of Laboratory Animals (National Academy Press, Washington, D.C., 1996).

\section{General Preparation}

Male New Zealand White rabbits weighing between 2.5 and $3.0 \mathrm{~kg}$ were anesthetized with intravenous sodium pentobarbital $(30 \mathrm{mg} / \mathrm{kg})$. Additional doses of pentobarbital were titrated as required to assure that pedal and palpebral reflexes were absent throughout the experiment. A tracheostomy was performed through a ventral midline incision, and the trachea was cannulated. The rabbits were ventilated (model 683, Harvard, Holliston, MA) with positive pressure using a room air-oxygen mixture $\left(\mathrm{FiO}_{2}=0.33\right)$. Arterial blood gas tensions and acid-base status were maintained within a normal physiologic range $\left(\mathrm{pH} 7.35-7.45, \mathrm{PaCO}_{2} 25-40 \mathrm{mmHg}\right.$, and $\mathrm{PaO}_{2} 90-150 \mathrm{mmHg}$ ) by adjusting the respiratory rate or tidal volume throughout the experiment. Body temperature was maintained with a heating blanket. Heparin- 


\begin{tabular}{|c|c|c|c|c|c|}
\hline \multirow[b]{2}{*}{ CONTROL } & \multicolumn{5}{|c|}{$\mathrm{MIN} \rightarrow-30 \mathrm{MIN} \rightarrow$} \\
\hline & BASELINE & & & & REPERFUSION \\
\hline \multirow[t]{2}{*}{ ISOFLURANE } & BASELINE & & $\begin{array}{l}\text { ISOFLURANE } \\
1.0 \text { MAC }\end{array}$ & MEMORY & REPERFUSION \\
\hline & \multicolumn{4}{|c|}{$\stackrel{\substack{2-\mathrm{MPG} \\
1 \mathrm{mg} / \mathrm{kg} / \min }}{2}$} & \\
\hline $\begin{array}{l}\text { ISOFLURANE + } \\
\text { 2-MPG }\end{array}$ & BASELINE & & $\begin{array}{l}\text { 1SOFLURANE } \\
\text { 1.0 MAC } \\
\end{array}$ & MEMORY & REPERFUSION \\
\hline \multirow[t]{2}{*}{$\begin{array}{l}\text { ISOFLURANE + } \\
\text { NAC }\end{array}$} & BASELINE & $\begin{array}{c}\mathrm{NAC} \\
150 \mathrm{mg} / \mathrm{kg} \\
\end{array}$ & $\begin{array}{l}\text { 1SOFLURANE } \\
\text { 1.0 MAC } \\
\end{array}$ & MEMORY & REPERFUSION \\
\hline & \multicolumn{4}{|c|}{$\longleftrightarrow \begin{array}{c}2-M P G \\
1 \mathrm{mg} / \mathrm{kg} / \min \end{array} \longrightarrow$} & \\
\hline 2-MPG & BASELINE & & & & REPERFUSION \\
\hline NAC & BASELINE & $\begin{array}{l}\mathrm{NAC} \\
150 \mathrm{mg} / \mathrm{kg}\end{array}$ & & & REPERFUSION \\
\hline
\end{tabular}

Fig. 1. Schematic illustration of the experimental protocol. ISO $=$ isoflurane; 2-MPG $=\boldsymbol{N}$-2-mercaptopropionyl glycine; $\mathrm{NAC}=\mathrm{N}$-acetylcysteine. filled catheters were inserted into the right carotid artery and the left jugular vein for measurement of arterial blood pressure and fluid or drug administration, respectively. Maintenance fluids consisted of $0.9 \%$ saline $\left(15 \mathrm{ml} \cdot \mathrm{kg}^{-1} \cdot \mathrm{h}^{-1}\right.$ ) that were continued for the duration of the experiment. A left thoracotomy was performed at the fourth intercostal space, and the heart was suspended in a pericardial cradle. A heparin-filled catheter was inserted into the left atria for the subsequent administration of dihydroethidium used to detect ROS production. A prominent branch of the left anterior descending coronary artery (LAD) was selected, and a silk ligature was placed around this artery approximately halfway between the base and apex for the production of coronary artery occlusion and reperfusion. Each rabbit was anticoagulated with $500 \mathrm{U}$ of heparin immediately before LAD occlusion. Coronary artery occlusion was verified by the presence of epicardial cyanosis and regional dyskinesia in the ischemic zone, and reperfusion was confirmed by observing an epicardial hyperemic response. Hemodynamics were continuously recorded on a polygraph (Grass model 7) throughout experimentation.

\section{Experimental Protocol}

The experimental design used in the present investigation is illustrated in figure 1 . Thirty minutes after instrumentation was completed and calibrated, baseline systemic hemodynamics were recorded. Rabbits were randomly assigned to one of six experimental groups using a Latin square design. All rabbits underwent a 30 min LAD occlusion followed by $3 \mathrm{~h}$ reperfusion. Rabbits received intravenous vehicle ( $0.9 \%$ saline), N-acetylcysteine (NAC; $150 \mathrm{mg} / \mathrm{kg}$ over $30 \mathrm{~min}$ ), ${ }^{18}$ or N-2-mercaptopropionyl glycine $\left(2-\mathrm{MPG} ; 1 \mathrm{mg} \cdot \mathrm{kg}^{-1} \cdot \min ^{-1}\right.$ over 75 $\mathrm{min})^{5}$ in the presence or absence of 1.0 minimum alveolar concentration (MAC) isoflurane. The end-tidal MAC value used for rabbits in the present investigation was $2.1 \% .{ }^{19}$ End-tidal concentrations of isoflurane were measured at the tip of the tracheostomy tube with an infra- red anesthetic analyzer that was calibrated with known standards before and during experimentation.

\section{Determination of Myocardial Infarct Size}

Myocardial infarct size was measured as previously described. ${ }^{20}$ Briefly, the left ventricular (LV) area at risk for infarction (AAR) was separated from surrounding normal areas, and the two regions were incubated at $37^{\circ} \mathrm{C}$ for 20 to $30 \mathrm{~min}$ in $1 \%$ 2,3,5-triphenyltetrazolium chloride in $0.1 \mathrm{~m}$ phosphate buffer adjusted to $\mathrm{pH} 7.4$. After overnight storage in 10\% formaldehyde, infarcted and noninfarcted myocardium within the AAR were carefully separated and weighed. Infarct size was expressed as a percentage of the AAR. Rabbits that developed intractable ventricular fibrillation and those with an AAR less than 15\% of LV mass were excluded from subsequent analysis.

\section{ROS Detection}

Dihydroethidium is oxidized by intracellular ROS to produce fluorescent ethidium that subsequently binds to DNA (Eth-DNA) further amplifying its fluorescence. ${ }^{21}$ The fluorescence observed after activation of the EthDNA complex is generally stable, but may be reduced by the presence of hydroxyl radicals. ${ }^{22,23}$ Thus, an increase in dihydroethidium oxidation to Eth-DNA and the subsequent increase in fluorescence are highly suggestive of superoxide anion generation. In two additional groups of rabbits $(\mathrm{n}=8)$, dihydroethidium $(2 \mathrm{mg})$ was rapidly injected into the left atrium $5 \mathrm{~min}$ before the administration of isoflurane (1 MAC) or at the corresponding time point in rabbits that were not subsequently exposed to the volatile anesthetic. Isoflurane was discontinued after $30 \mathrm{~min}$, and the rabbits were euthanized after $1 \mathrm{~h}$ with a lethal dose of pentobarbital. The heart was rapidly excised. The LV was isolated, divided into four sections of equal size, and frozen in liquid nitrogen. Kryostat sections $(20 \mu \mathrm{m})$ of the LV were mounted on standard microscope slides. Using a laser fluorescence 
Table 1. Systemic Hemodynamics

\begin{tabular}{|c|c|c|c|c|c|c|c|}
\hline & \multirow[b]{2}{*}{ Baseline } & \multirow[b]{2}{*}{ Isoflurane } & \multirow[b]{2}{*}{ Preocclusion } & \multirow[b]{2}{*}{30 min $\mathrm{CAO}$} & \multicolumn{3}{|c|}{ Reperfusion (h) } \\
\hline & & & & & 1 & 2 & 3 \\
\hline \multicolumn{8}{|l|}{$\mathrm{HR}\left(\min ^{-1}\right)$} \\
\hline CON & $259 \pm 11$ & - & $266 \pm 11$ & $264 \pm 11$ & $260 \pm 10$ & $265 \pm 10$ & $268 \pm 12$ \\
\hline ISO & $260 \pm 10$ & $273 \pm 8$ & $260 \pm 5$ & $240 \pm 9^{*}$ & $242 \pm 9^{\star}$ & $235 \pm 10^{*}$ & $238 \pm 9^{*}$ \\
\hline ISO+2-MPG & $255 \pm 9$ & $282 \pm 6^{*}$ & $269 \pm 7$ & $275 \pm 13^{\star}$ & $249 \pm 8$ & $241 \pm 6$ & $242 \pm 2$ \\
\hline ISO+ NAC & $251 \pm 7$ & $276 \pm 7^{*}$ & $275 \pm 6^{*}$ & $270 \pm 8$ & $265 \pm 8$ & $264 \pm 4$ & $270 \pm 7$ \\
\hline 2-MPG & $243 \pm 9$ & - & $259 \pm 8$ & $263 \pm 13$ & $234 \pm 5$ & $230 \pm 9$ & $236 \pm 12$ \\
\hline NAC & $255 \pm 9$ & - & $261 \pm 7$ & $255 \pm 11$ & $248 \pm 11$ & $251 \pm 13$ & $247 \pm 13$ \\
\hline \multicolumn{8}{|l|}{ MAP (mmHg) } \\
\hline $\mathrm{CON}$ & $88 \pm 4$ & - & $85 \pm 3$ & $75 \pm 2^{*}$ & $83 \pm 4$ & $81 \pm 6$ & $78 \pm 5^{\star}$ \\
\hline ISO & $89 \pm 3$ & $67 \pm 5^{\star}$ & $82 \pm 3$ & $73 \pm 4^{*}$ & $74 \pm 5^{\star}$ & $77 \pm 4^{*}$ & $79 \pm 4^{*}$ \\
\hline ISO + 2-MPG & $89 \pm 2$ & $51 \pm 5^{\star}$ & $79 \pm 4$ & $68 \pm 6^{*}$ & $75 \pm 6$ & $76 \pm 5$ & $74 \pm 4$ \\
\hline ISO + NAC & $90 \pm 4$ & $62 \pm 2^{\star}$ & $82 \pm 4$ & $77 \pm 4^{*}$ & $82 \pm 5$ & $83 \pm 5$ & $83 \pm 3$ \\
\hline 2-MPG & $90 \pm 3$ & - & $85 \pm 2$ & $69 \pm 6^{*}$ & $72 \pm 6^{*}$ & $74 \pm 7^{\star}$ & $74 \pm 7^{*}$ \\
\hline \multirow{2}{*}{\multicolumn{8}{|c|}{$\mathrm{RPP}\left(\mathrm{bpm} \cdot \mathrm{mmHg} \cdot 10^{-3}\right)$}} \\
\hline & & & & & & & \\
\hline CON & $26.3 \pm 1.9$ & - & $26.6 \pm 1.6$ & $23.0 \pm 1.5^{\star}$ & $25.0 \pm 2.1$ & $24.7 \pm 2.3$ & $23.6 \pm 2.3^{*}$ \\
\hline ISO & $26.3 \pm 1.5$ & $22.8 \pm 1.4^{*}$ & $25.3 \pm 1.0$ & $20.5 \pm 1.3^{*}$ & $20.9 \pm 1.8^{*}$ & $20.8 \pm 1.4^{\star}$ & $21.5 \pm 1.6^{*}$ \\
\hline ISO + 2-MPG & $25.7 \pm 1.0$ & $20.4 \pm 1.7^{*}$ & $25.6 \pm 1.0$ & $22.0 \pm 1.6^{\star}$ & $21.8 \pm 1.9^{*}$ & $20.7 \pm 1.6^{\star}$ & $20.2 \pm 0.9^{*}$ \\
\hline ISO + NAC & $26.0 \pm 1.4$ & $23.2 \pm 1.1$ & $27.0 \pm 1.3$ & $24.3 \pm 1.6$ & $24.8 \pm 1.4$ & $24.9 \pm 1.4$ & $25.5 \pm 0.7$ \\
\hline 2-MPG & $24.4 \pm 0.9$ & - & $25.3 \pm 1.1$ & $20.5 \pm 1.6^{\star}$ & $18.9 \pm 1.2^{*}$ & $19.2 \pm 1.8^{*}$ & $19.8 \pm 1.7^{*}$ \\
\hline NAC & $25.0 \pm 1.6$ & - & $25.3 \pm 1.1$ & $21.8 \pm 1.4^{\star}$ & $21.2 \pm 1.4^{*}$ & $21.8 \pm 1.9^{*}$ & $21.7 \pm 1.9^{\star}$ \\
\hline
\end{tabular}

Data are mean \pm SEM.

* Significantly $(P<0.05)$ different from baseline.

$\mathrm{CAO}=$ coronary artery occlusion; $\mathrm{HR}=$ heart rate; MAP = mean arterial blood pressure; $\mathrm{RPP}=$ rate-pressure product; CON = control; ISO = isoflurane; 2-MPG $=\mathrm{N}$-2-mercaptopropionyl glycine; NAC $=\mathrm{N}$-acetylcysteine.

imaging system (MRC 600 Laser Scanning Confocal Microscopic Imaging System; Bio-Rad Laboratories; Philadelphia, PA) mounted on a Microscope (Optiphot; Nikon Corporation; Tokyo, Japan) images were recorded and stored for subsequent offline analysis on a computer workstation equipped with image analysis software (MetaMorph; Universal Imaging Corporation; Downingtown, PA). Use of the $40 \times$ objective yielded a $400 \times$ end magnification on a $292 \times 195 \mu \mathrm{m}^{2}$ digital image $(768 \times$ 512 pixels). The signal-to-noise ratio was enhanced using the Kalman method. Excitation was produced using a Krypton-Argon laser at a wavelength of $488 \mathrm{~nm}$, and emitted fluorescence measured at $550 \mathrm{~nm}$ after long pass filtering. The pixel intensity of each myocyte nucleus was determined. Background was identified as an area without cells or with minimal cytosol fluorescence. In each rabbit, 20 Kalman-averaged images were obtained and approximately 6 to 8 dihydroethidium-stained myocardial cells were analyzed by subtraction of background fluorescence from the pixel intensity of the myocardial nuclei.

\section{Statistical Analysis}

Statistical analysis of data within and between groups was performed with analysis of variance (ANOVA) for repeated measures followed by Student Newman-Keuls test. Changes within and between groups were considered statistically significant when the $P$ value was less than 0.05 . Student $t$ test was used to compare differences of pixel intensities in ROS detection experiments.
Statistical significance was defined as $P<0.05$. All data are expressed as mean $\pm \mathrm{SEM}$.

\section{Results}

Sixty-one rabbits were instrumented to obtain 48 successful myocardial infarct size experiments. Eight rabbits were excluded because the AAR/LV did not exceed 15\% ( 1 control; 2 isoflurane alone; 1 isoflurane +2 -MPG; 3 isoflurane $+\mathrm{NAC}$; and 1 NAC alone). Five rabbits were excluded because of intractable ventricular fibrillation (1 control; 2 isoflurane alone; 1 isoflurane $+2-\mathrm{MPG}$; and 1 2-MPG alone).

\section{Systemic Hemodynamics}

No differences in hemodynamics were observed between experimental groups under control conditions (table 1). Isoflurane significantly $(P<0.05)$ decreased mean arterial pressure and rate-pressure product in the presence or absence of ROS scavengers. Hemodynamics returned to baseline values $15 \mathrm{~min}$ after isoflurane had been discontinued. Coronary artery occlusion and reperfusion produced similar decreases in mean arterial pressure and rate-pressure product in each experimental group.

\section{Myocardial Infarct Size}

The body weight, LV weight, AAR weight, and AAR/LV were similar between groups (table 2). Isoflurane reduced myocardial infarct size $(24 \pm 4 \%$ of the AAR; $n=$ 
Table 2. Area at Risk

\begin{tabular}{lcccc}
\hline & $\begin{array}{c}\text { Body Weight } \\
(\mathrm{g})\end{array}$ & LV WT (g) & AAR WT (g) & $\begin{array}{c}\text { AAR/LV } \\
(\%)\end{array}$ \\
\hline CON & $2960 \pm 50$ & $3.16 \pm 0.11$ & $0.94 \pm 0.13$ & $30 \pm 4$ \\
ISO & $3050 \pm 80$ & $3.16 \pm 0.10$ & $1.04 \pm 0.09$ & $33 \pm 3$ \\
ISO + 2-MPG & $3000 \pm 70$ & $3.10 \pm 0.13$ & $1.04 \pm 0.13$ & $33 \pm 4$ \\
ISO + NAC & $2780 \pm 50$ & $2.99 \pm 0.11$ & $0.88 \pm 0.10$ & $29 \pm 3$ \\
2-MPG & $2900 \pm 40$ & $3.10 \pm 0.08$ & $1.08 \pm 0.12$ & $34 \pm 3$ \\
NAC & $2790 \pm 80$ & $2.88 \pm 0.08$ & $0.83 \pm 0.07$ & $29 \pm 3$ \\
\hline
\end{tabular}

Data are mean \pm SEM.

$\mathrm{LV}=$ left ventricle; $\mathrm{WT}=$ weight; $\mathrm{AAR}=$ area at risk; $\mathrm{CON}=$ control; ISO $=$ isoflurane; 2-MPG $=\mathrm{N}$-2-mercaptopropionyl glycine; NAC $=\mathrm{N}$-acetylcysteine.

10; fig. 2) as compared to control experiments ( $43 \pm 3 \%$; $\mathrm{n}=8$ ). NAC and 2-MPG alone did not affect infarct size ( $47 \pm 3 \% ; n=8$ and $46 \pm 3 \% ; n=7$, respectively), but these ROS scavengers blocked the protective effects of isoflurane $(43 \pm 3 \% ; \mathrm{n}=7$ and $42 \pm 5 \% ; \mathrm{n}=8$, respectively).

\section{Reactive Oxygen Species Production}

Ethidium-DNA fluorescence was detected in all images examined (fig. 3). Rabbits pretreated with 1.0 MAC isoflurane demonstrated enhanced fluorescence in myocardial nuclei compared with the cytosol, in contrast to the findings in rabbits that did not receive the volatile agent (fig. 4). Fluorescence intensity in myocardial nuclei was significantly greater $(28 \pm 12$ vs. $-6 \pm 9$ fluorescence units) in isoflurane-pretreated compared with untreated rabbits.

\section{Discussion}

Mitochondria have been shown to produce small quantities of ROS during brief periods of ischemia that cause preconditioning., ${ }^{5,6}$ The protective effects of the selective mitochondrial $\mathrm{K}_{\mathrm{ATP}}$ channel opener, diazoxide, were blocked by pretreatment with ROS scavengers in isolated rabbit hearts. ${ }^{24}$ Diazoxide also increased oxidation of the ROS probe mitotracker orange in vitro, an action that was attenuated by pretreatment with the selective mitochondrial $\mathrm{K}_{\mathrm{ATP}}$ channel antagonist 5-hydroxydecanoate (5-HD) or the ROS scavengers NAC and 2-MPG. ${ }^{25}$ Diazoxide directly increased ROS production as measured by the hydrogen peroxide probe $2^{\prime}, 7^{\prime}$-dichlorofluorescein diacetate ${ }^{26}$ in rat ventricular myocytes and isolated hearts. Whether volatile anesthetics increase ROS production or modulate the actions of oxygen-derived free radicals as potential mechanisms by which these agents produce myocardial protection remains unclear. Sevoflurane has been shown to impair endothelium-dependent relaxation of canine mesenteric arteries by an oxygen-derived free radical mechanism. ${ }^{27}$ Isoflurane reduced hydroxyl radical production in the ischemic rat heart. ${ }^{28}$ In contrast, Müllenheim et al. ${ }^{17}$ very recently demonstrated that 2-MPG and another ROS scavenger Mn(III)tetrakis(4-benzoic acid)porphyrine chloride abolished the protective effect of isoflurane in rabbit hearts. These data were the first to implicate a role for ROS in anesthetic-induced preconditioning, and suggested that volatile agents may be capable of producing small quantities of ROS that serve as mediators of cardioprotection.

The results of the present investigation confirm and extend the findings of Müllenheim et al. ${ }^{17}$ and indicate that the ROS scavengers NAC and 2-MPG blocked the reduction in myocardial infarct size produced by isoflurane when these drugs were administered during exposure to isoflurane. The results further demonstrate that intensity of Eth-DNA fluorescence measured with confocal microscopy is significantly increased during a $30 \mathrm{~min}$ pretreatment with 1.0 MAC isoflurane. These data indicate that isoflurane directly increases superoxide anion generation independent of coronary artery occlusion and reperfusion and strongly imply that this ROS production mediates protection against irreversible ischemic injury. Mitochondria are a likely source for the production of the superoxide anion. ${ }^{29}$ Superoxide anion generated experimentally from the enzyme complex hypoxanthine-xanthine oxidase mimics the protective effects of preconditioning. ${ }^{30}$ Thus, it appears likely that superoxide anion produced by mitochondria may activate intracellular signaling responsible for the protective effect of isoflurane. However, we did not specifically determine the source of ROS production by isoflurane or whether the generation of this oxygen-derived free radical species is linked to mitochondrial $\mathrm{K}_{\mathrm{ATP}}$ channel opening in the present investigation. These objectives are important goals of future research.

The specific oxygen-derived free radicals responsible for activation of the signal transduction of preconditioning are unknown. The $\mathrm{Cu}, \mathrm{Zn}$-superoxide dismutase (SOD) inhibitor diethyldithiocarbamic acid is a selective inhibitor of the cytosolic conversion of superoxide anion

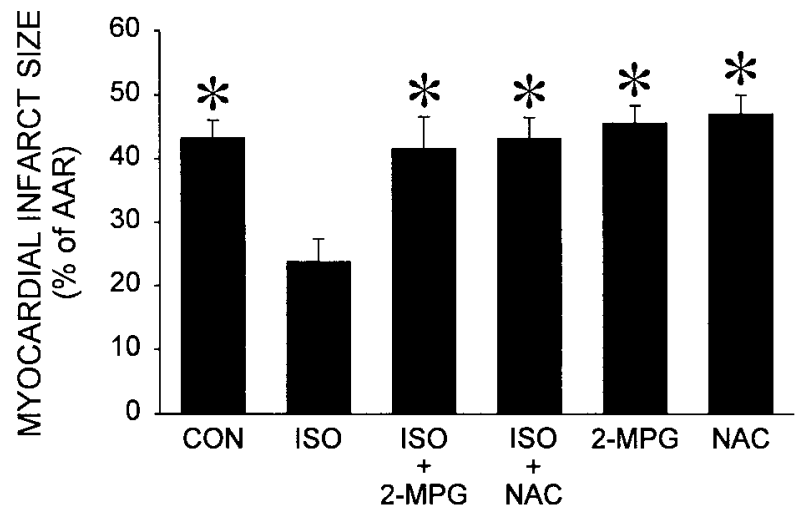

Fig. 2. Myocardial infarct size expressed as a percentage of the left ventricular area at risk (AAR) in rabbits receiving saline (CON), N-2-mercaptopropionyl glycine (2-MPG), or $\mathbf{N}$-acetylcysteine (NAC) in the presence and absence of 1.0 MAC isoflurane (ISO). *Significantly $(P<0.05)$ different from ISO. 


\section{A}

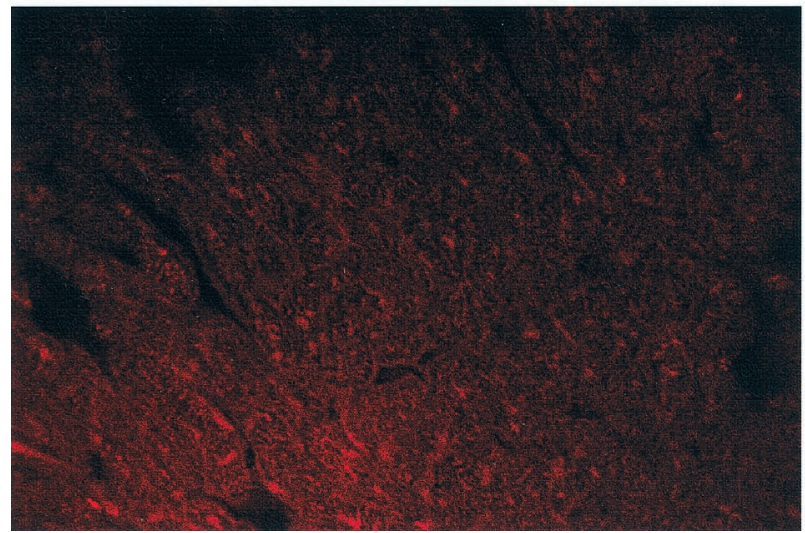

B

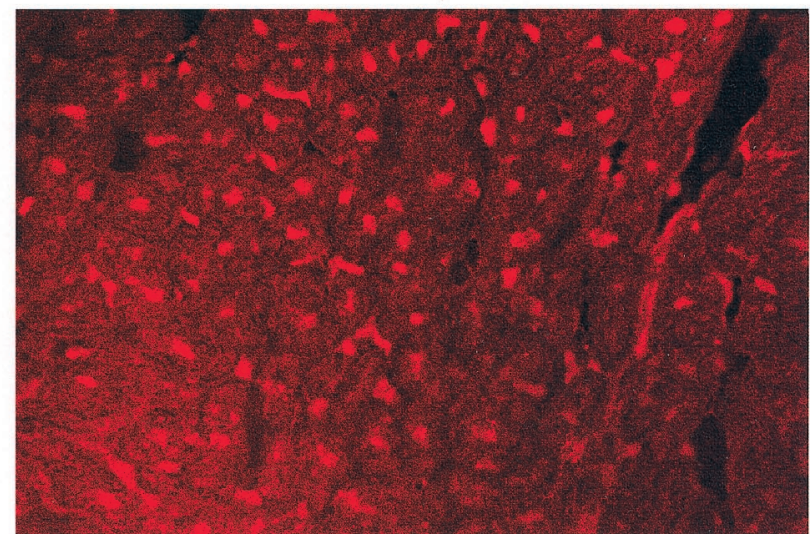

Fig. 3. Representative photomicrographs demonstrating enhanced production of superoxide anion by the expression of fluorescent ethidium bound to nuclear DNA. The fluorescence in myocardial nuclei in rabbits treated with 1.0 MAC isoflurane for 30 min (B) was more intense than that observed in rabbits that were not exposed to isoflurane $(A)$.

to hydrogen peroxide and abolished protection produced by hypoxic preconditioning in embryonic cardiac myocytes. ${ }^{6}$ These results suggested that conversion of superoxide anion to hydrogen peroxide may be an important step for oxidant induction of hypoxic preconditioning. $\mathrm{N}$-acetylcysteine is a sulfhydryl-containing glutathione precursor that exerts antioxidant effects by contributing to glutathione synthesis, serving as a glutathione peroxidase substrate, and directly scavenging several oxygen-derived free radical species primarily by the actions of reduced glutathione. ${ }^{31}$ Mitochondria contain large quantities of SOD, and the vast majority of superoxide anion generated as a consequence of mitochondrial electron transport is enzymatically dismutated to hydrogen peroxide and oxygen by mitochondrial MnSOD and cytosolic $\mathrm{Cu}, \mathrm{Zn}$-SOD. Glutathione peroxidase contained in the cytosol subsequently catalyzes the reduction of hydrogen peroxide to water. Reduced glutathione acts as the electron donor of glutathione peroxidase in this reaction. Thus, NAC maintains the cytosolic concentration of reduced glutathione and facilitates metabolism of hydrogen peroxide produced by the univalent reduction of superoxide anion. 2-MPG also acts as a sulfhydryl donor to glutathione peroxidase, and several studies indicate that 2-MPG may be more specific for mitochondrial activity than NAC. ${ }^{32-34}$ We did not specifically identify the particular ROS scavenged by NAC or 2-MPG that was responsible for isoflurane-induced preconditioning. However, the present results indicate that exposure to isoflurane is accompanied by the generation of superoxide anion in ventricular myocytes, most likely in the mitochondria, in the absence of ischemia and reperfusion. Thus, it appears likely that the superoxide anion or another ROS generated by the superoxide anion dismutation pathway is involved in the signal transduction of isoflurane-induced preconditioning.
The present results should be interpreted within the constraints of several potential limitations. The area of the left ventricle at risk for infarction and coronary collateral blood flow are important determinants of the extent of myocardial infarction. The AAR was similar between experimental groups. Minimal coronary collateral blood flow has been previously reported in rabbits. ${ }^{35}$ Thus, it is unlikely that the present results were affected by the size of the AAR or magnitude of coronary collateral blood flow.

Isoflurane caused similar hemodynamic effects in the presence or absence of ROS scavengers, and there were no differences in hemodynamics between groups after discontinuation of isoflurane. Thus, the present results occurred independent of the hemodynamic effects of isoflurane and/or the ROS scavengers. Nevertheless, coronary venous oxygen tension and myocardial oxygen consumption were not directly quantified in the present investigation, and differences in myocardial oxygen me-

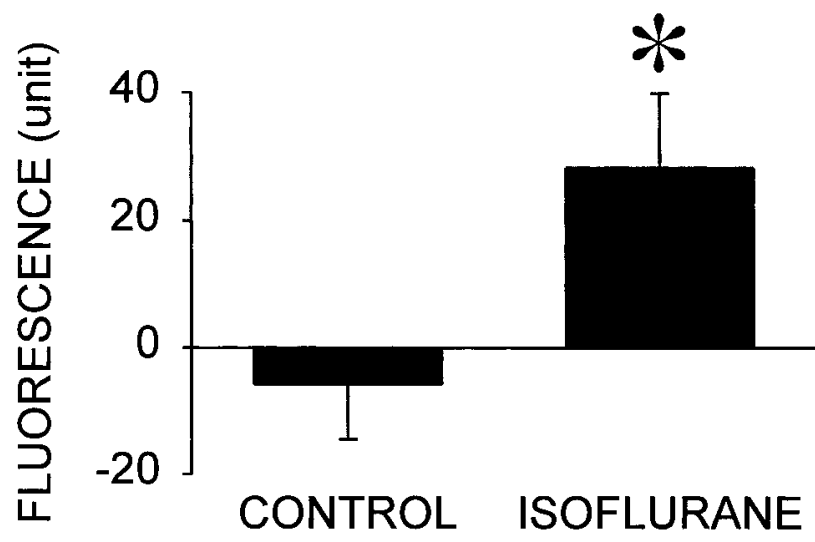

Fig. 4. Histogram depicting the effects of isoflurane on superoxide anion production measured using dihydroethidium staining. Fluorescence of ethidium bound to nuclear DNA was significantly $(* P<0.05)$ increased by administration of isoflurane. 
tabolism during the administration of isoflurane with or without ROS scavengers cannot be completely excluded from the analysis. However, no differences in primary hemodynamic determinants of myocardial oxygen consumption were observed, and an indirect indicator (i.e., rate-pressure product) of myocardial oxygen consumption was also similar between experimental groups.

Experiments using dihydroethidium as an indicator of superoxide anion production provide primarily qualitative results. Dihydroethidium can catalyze the dismutation of superoxide anion, thus, rates of superoxide anion production may be underestimated using this technique. ${ }^{36}$ In addition, cytochrome $c$ can oxidize dihydroethidium. ${ }^{36}$ It is unlikely that cytochrome $c$ release occurred in experiments during which dihydroethidium was used because animals were not subjected to ischemia and reperfusion. Isoflurane is unlikely to cause cytochrome $c$ release because this anesthetic has previously been demonstrated to decrease apoptosis and inhibit caspase activation in ventricular myocytes. ${ }^{37}$

In summary, the present results provide direct evidence that isoflurane generates ROS in rabbit ventricular myocardium in vivo using a laser fluorescence confocal microscopic imaging technique. The present findings further indicate that scavenging of these ROS by NAC and 2-MPG abolishes myocardial protection produced by isoflurane. These findings suggest that generation of ROS by volatile anesthetics is an essential part of the signaling pathway of anesthetic-induced preconditioning.

The authors thank David Schwabe, B.S.E.E. for technical assistance, and Mary Lorence-Hanke, A.A. (both from the Department of Anesthesiology, Medical College of Wisconsin, Milwaukee, Wisconsin) for assistance in preparation of the manuscript.

\section{References}

1. Zweier JL, Flaherty JT, Weisfeldt ML: Direct measurement of free radical generation following reperfusion of ischemic myocardium. Proc Natl Acad Sci USA $1987 ; 84: 1404-7$

2. Bolli R, Patel BS, Jeroudi MO, Lai EK, McCay PB: Demonstration of free radical generation in "stunned" myocardium of intact dogs with the use of the spin trap alpha-phenyl N-tert-butyl nitrone. J Clin Invest 1988; 82:476-85

3. Ambrosio G, Zweier JL, Duilio C, Kuppusamy P, Santoro G, Elia PP, Tritto I, Cirillo P, Condorelli M, Chiariello M, Flaherty JT: Evidence that mitochondrial respiration is a source of potentially toxic oxygen free radicals in intact rabbit hearts subjected to ischemia and reflow. J Biol Chem 1993; 268:18532-41

4. Ambrosio G, Tritto I, Chiariello $M$ : The role of oxygen free radicals in preconditioning. J Mol Cell Cardiol 1995; 27:1035-9

5. Baines CP, Goto M, Downey JM: Oxygen radicals released during ischemic preconditioning contribute to cardioprotection in the rabbit myocardium. J Mol Cell Cardiol 1997; 29:207-16

6. Vanden Hoek TL, Becker LB, Shao Z, Li C, Schumacker PT: Reactive oxygen species released from mitochondria during brief hypoxia induce preconditioning in cardiomyocytes. J Biol Chem 1998; 273:18092-8

7. Tanaka M, Fujiwara H, Yamasaki K, Sasayama S: Superoxide dismutase and $\mathrm{N}$-2-mercaptopropionyl glycine attenuate infarct size limitation effect of ischaemic preconditioning in the rabbit. Cardiovasc Res 1994; 28:980 -6

8. Kersten JR, Orth KG, Pagel PS, Mei DA, Gross GJ, Warltier DC: Role of adenosine in isoflurane-induced cardioprotection. ANESTHEsIology 1997; 86:1128-39

9. Cope DK, Impastato WK, Cohen MV, Downey JM: Volatile anesthetics protect the ischemic rabbit myocardium from infarction. ANESTHESIOLOGY 1997; 86:699-709

10. Roscoe AK, Christensen JD, Lynch C, III: Isoflurane, but not halothane, induces protection of human myocardium via adenosine A1 receptors and adenosine triphosphate-sensitive potassium channels. ANesthesIology 2000; 92: $1692-701$

11. Toller WG, Montgomery MW, Pagel PS, Hettrick DA, Warltier DC, Kersten JR: Isoflurane-enhanced recovery of canine stunned myocardium: Role for protein kinase C? Anesthesiology 1999; 91:713-22

12. Toller WG, Kersten JR, Gross ER, Pagel PS, Warltier DC: Isoflurane preconditions myocardium against infarction via activation of inhibitory guanine (Gi) nucleotide binding proteins. ANESTHESIOLOGY 2000; 92:1400-7

13. Kersten JR, Schmeling TJ, Pagel PS, Gross GJ, Warltier DC: Isoflurane mimics ischemic preconditioning via activation of $\mathrm{K}_{\mathrm{ATP}}$ channels. Reduction of myocardial infarct size with an acute memory phase. AnesthesIology 1997; 87:361-70

14. Toller WG, Gross ER, Kersten JR, Pagel PS, Gross GJ, Warltier DC: Sarcolemmal and mitochondrial adenosine triphosphate-dependent potassium $\left(\mathrm{K}_{\mathrm{ATP}}\right)$ channels. Mechanism of desflurane-induced cardioprotection. ANESTHESIOLOGY 2000; 92:1731-9

15. Ismaeil MS, Tkachenko I, Gamperl AK, Hickey RF, Cason BA: Mechanisms of isoflurane-induced myocardial preconditioning in rabbits. ANESTHESIOLOGY 1999; 90:812-21

16. Piriou V, Chiari P, Knezynski S, Bastien O, Loufoua J, Lehot JJ, Foex P, Annat G, Ovize M: Prevention of isoflurane-induced preconditioning by 5-hydroxydecanoate and gadolinium: Possible involvement of mitochondrial adenosine triphosphate-sensitive potassium and stretch-activated channels. ANESTHESOLOGY 2000; 93:756-64

17. Müllenheim J, Ebel D, FräBdorf J, Preckel B, Thämer V, Schlack W: Isoflurane preconditions myocardium against infarction via release of free radicals. ANESTHESIOLOGY 2002; 96:934-40

18. Ferrari R, Ceconi C, Curello S, Cargnoni A, Alfieri O, Pardini A, Marzollo P, Visioli O: Oxygen free radicals and myocardial damage: Protective role of thiolcontaining agents. Am J Med 1991; 91:95S-105S

19. Drummond JC: MAC for halothane, enflurane, and isoflurane in the New Zealand white rabbit: And a test for the validity of MAC determinations. ANESTHESIOLOGY $1985 ; 62: 336-8$

20. Warltier DC, Zyvoloski MG, Gross GJ, Hardman HF, Brooks HL: Determination of experimental myocardial infarct size. J Pharmacol Methods 1981; 6:199-210

21. Carter WO, Narayanan PK, Robinson JP: Intracellular hydrogen peroxide and superoxide anion detection in endothelial cells. J Leukoc Biol 1994; 55:253-8

22. Prutz WA: Inhibition of DNA-ethidium bromide intercalation due to free radical attack upon DNA. I. Comparison of the effects of various radicals. Radiat Environ Biophys 1984; 23:1-6

23. Prutz WA: Inhibition of DNA-ethidium bromide intercalation due to free radical attack upon DNA. II. Copper(II)-catalysed DNA damage by $\mathrm{O}_{2}$. Radiat Environ Biophys 1984; 23:7-18

24. Pain T, Yang XM, Critz SD, Yue Y, Nakano A, Liu GS, Heusch G, Cohen MV, Downey JM: Opening of mitochondrial K(ATP) channels triggers the preconditioned state by generating free radicals. Circ Res 2000; 87:460-6

25. Carroll R, Gant VA, Yellon DM: Mitochondrial K(ATP) channel opening protects a human atrial-derived cell line by a mechanism involving free radical generation. Cardiovasc Res 2001; 51:691-700

26. Forbes RA, Steenbergen C, Murphy E: Diazoxide-induced cardioprotection requires signaling through a redox- sensitive mechanism. Circ Res 2001; 88:802-9

27. Yoshida K, Okabe E: Selective impairment of endothelium-dependent relaxation by sevoflurane: Oxygen free radicals participation. ANESTHESIOLOGY 1992; 76:440 -7

28. Nakamura $\mathrm{T}$, Kashimoto $\mathrm{S}$, Oguchi $\mathrm{T}$, Kumazawa $\mathrm{T}$ : Hydroxyl radical formation during inhalation anesthesia in the reperfused working rat heart. Can J Anaesth 1999; 46:470 -5

29. Becker LB, vanden Hoek TL, Shao ZH, Li CQ, Schumacker PT: Generation of superoxide in cardiomyocytes during ischemia before reperfusion. Am J Physiol Heart Circ Physiol 1999; 277:H2240-6

30. Tritto I, D'Andrea D, Eramo N, Scognamiglio A, De Simone C, Violante A, Esposito A, Chiariello M, Ambrosio G: Oxygen radicals can induce preconditioning in rabbit hearts. Circ Res 1997; 80:743-8

31. Aruoma OI, Halliwell B, Hoey BM, Butler J: The antioxidant action of $\mathrm{N}$-acetylcysteine: its reaction with hydrogen peroxide, hydroxyl radical, superoxide, and hypochlorous acid. Free Radic Biol Med 1989; 6:593-7

32. Beyersdorf F, Zimmer G, Fuchs J, Kraft H, Veit P, Satter P: Improvement of myocardial function after global hypoxia by protection of the inner mitochondrial membrane. Arzneimittelforschung 1987; 37:142-9

33. Beyersdorf F, Fuchs J, Eberhardt B, Stauder M, Satter P, Zimmer G: Myocardial protection by 2-mercaptopropionylglycine during global ischemia in dogs. Arzneimittelforschung 1989; 39:46-9

34. Fuchs J, Mainka L, Zimmer G: 2-Mercaptopropionylglycine and related compounds in treatment of mitochondrial dysfunction and postischemic myocardial damage. Arzneimittelforschung 1985; 35:1394-402

35. Maxwell MP, Hearse DJ, Yellon DM: Species variation in the coronary collateral circulation during regional myocardial ischaemia: A critical determinant of the rate of evolution and extent of myocardial infarction. Cardiovasc Res 1987; 21:737- 46

36. Benov L, Sztejnberg L, Fridovich I: Critical evaluation of the use of hydroethidine as a measure of superoxide anion radical. Free Radic Biol Med 1998; 25:826-31

37. Zaugg M, Jamali NZ, Lucchinetti E, Shafiq SA, Siddiqui MA: Norepinephrine-induced apoptosis is inhibited in adult rat ventricular myocytes exposed to volatile anesthetics. AnEsThesiology 2000; 93:209-18 